\begin{abstract}
FE DE ERRATAS
Por error tipográfico, en el artículo Valor pronóstico del parámetro inmunohistoquímico p53 en los estudios I y II del carcinoma epidermoide de lengua móvil (Revista Española de Cirugía Oral y Maxilofacial 2003; 5:280-7), se omitió su discusión, por lo que se publica ahora.
\end{abstract}

\section{Valor pronóstico del parámetro inmunohisto- químico p53 en los estadios I y II del carcinoma epidermoide de lengua móvil}

Los autores presentan un interesante estudio sobre el valor pronóstico de la p53 en el carcinoma lingual. Indudablemente, la búsqueda de factores pronósticos mesurables y fiables constituye un objetivo básico en la investigación del cáncer y puede sentar las bases para la realización de tratamientos más o menos agresivos.

Los factores pronósticos en cáncer de cabeza y cuello son de distinta índole. Dentro de los factores pronósticos generales tenemos edad, infección postoperatoria, inmunidad, transfusiones sanguíneas y tiempo de inicio de la radioterapia. Dentro de los factores clínicos se ha valorado la localización tumoral, tamaño tumoral, existencia de adenopatías cervicales, metástasis a distancia, metástasis cutáneas, estadio TNM, y tiempo de recurrencia. Como factores histológicos pronósticos se han estudiado la morfología de invasión del frente tumoral, infiltrado inflamatorio peritumoral, grado de diferenciación, tamaño del núcleo celular, índice de mitosis, capacidad de clonación celular in vitro, infiltración perineural, invasión microvascular, angiogénesis tumoral, espesor tumoral, afectación tumoral de la arteria carótida intema, margen de resección tumoral afecto, margen de resección afecto en la disección cervical y sistemas como el de Jakobsson que utiliza combinación de distintos factores histológicos. Muchos de estos factores son expresión de alteraciones moleculares fruto de modificaciones en el contenido de los genes, bien sea por traslocación, mutación o sobreexpresión. Los conocimientos en las bases genéticas del carcinoma epidermoide de cavidad oral no han sido tan importantes como en otros tumores. ${ }^{1,2,19}$ Los avances futuros en este terreno serán importantes para el diseño de nuevas altemativas terapéuticas y predicción del pronóstico del paciente.

Las alteraciones genéticas descritas en los carcinomas de cabeza y cuello son numerosas: mutaciones de la p53, amplificación de bcl-1, c-erbB1 y c-myc, coamplificación de int-2/bcl-1 e int-2/c-erbB-1.3,19 En un 75\% de los carcinomas se encuentra elevado alguno de los siguientes marcadores tumorales, CEA, CA 19-9, SCC,
TK, DTTPASE, pero ninguno de ellos se correlaciona con el estadio, la extensión metastásica o el pronóstico, siendo además sus modificaciones postratamiento erráticas y poco predecibles, pudiendo existir elevación sin recidiva. ${ }^{4}$ La región cromosómica 11q13 es un lugar crítico para la traslocación de los oncogenes bcl-1, PRAD-1/ciclina D1 y el gen del factor de crecimiento del fibroblasto HST1. La amplificación entre 2 y 10 veces del locus 11q13 se correlaciona con un patrón de infiltración más difuso del tumor y peor grado de diferenciación pero sin implicación pronóstica. ${ }^{5}$ La asociación entre metástasis y el factor de crecimiento endotelial en sus isoformas A y C es muy significativa, no obstante su asociación pronóstica no ha podido constatarse. ${ }^{6,7}$ Aquellos pacientes con niveles elevados pretratamiento del péptido angiogénico bFGF, presentan ligero peor pronóstico que aquellos individuos con niveles normales, sin embargo, factores como la metaloproteinasa 2 matrix no presentan correlación con el pronóstico.7

La Ciclina D1 pertenece a la familia de ciclinas que actúan sobre las ciclinas quinasas, responsables de la regulación de la transición de las distintas fases del ciclo celular. La sobreexpresión o amplificación de la Ciclina D1 está presente entre el 26 y 39\% de los carcinomas de cabeza y cuello, y tiene relación con el consumo de alcohol y tabaco. La sobreexpresión tiene correlación con el pronóstico y forma independiente, siendo peor cuanto mayor es la sobreexpresión de la Ciclina D1, tanto en carcinomas orales, laríngeos o de cabeza y cuello en general. $8-10$

El Syndecan-1 está relacionado con la maduración y diferenciación queratinocítica de los epitelios y se produce una reducción de su producción en los carcinomas epidermoides. Unos niveles elevados del Syndecan-1 se correlacionan con un buen pronóstico, ${ }^{11} \mathrm{e}$ igualmente se asocia con tumores más pequeños y mejor grado de diferenciación. ${ }^{12}$

La interleuquina 2 es posible medirla en sangre, sus niveles séricos se relacionan con el pronóstico de los carcinomas epidermoides siendo un factor independiente de otros y el más significativo en la serie de Tartour. ${ }^{13}$ 
La familia de la proteína quinasa $\mathrm{C}$ ha sido relacionada con el desarrollo del crecimiento celular y la tumorogénesis. Nosotros hemos podido demostrar una clara sobreexpresión en los carcinomas epidermoides de cabeza y cuello del isotipo عPKC, pero sin correlación pronóstica. Sin embargo, el isotipo ePKC es un potente factor pronóstico, de tal manera que los pacientes con niveles elevados o normales tienen una tasa de recurrencia del $25 \%$ frente $85 \%$ de recidiva de los pacientes que tienen disminuida la expresión de la $\varepsilon$ PKC. Lo cual significa que la $\varepsilon$ PKC tiene un carácter protector y en nuestra serie fue el factor más significativo. ${ }^{14}$

La p53 es una fosfoproteína nuclear que participa en la regulación de la transcripción génica, síntesis y reparación del DNA, coordinación del ciclo celular y la apoptosis. Las alteraciones en la función de la p53 pueden ser por mutación o secuestro por proteínas celulares tales como mdm2 o la oncoproteína del papilomavirus E6. Las mutacionas pueden ser debidas a mutaciones puntuales, delección o inserción, resultado en la producción de una proteína anómala o no producción de la misma. Usar la técnica de inmunohistoquímica para la detención de la p53 presenta ciertos problemas que se deben conocer. La p53 es una proteína de vida media corta, 20 minutos, y por ello en condiciones normales no se detecta. Normalmente cuando se detecta una proteína p53 es una proteína mutada que es anormalmente estable y se acumula. Sin embargo, existen mutaciones que no desarrollan esta acumulación y por ende no son detectables. Así mismo, la sobre expresión de «wild type»p53 puede tener una marcada tinción inmunohistoquímica. Todas estas circunstancias hacen muy difícil la interpretación por inmunohistoquímica de la p53 y, fiel reflejo de ello, es la amplia discordancia en los trabajos publicados sobre la p53 y el pronóstico en el carcinoma epidermoide de cabeza y cuello. Para evitar estas discordancias lo ideal sería secuenciar la p53 y comprobar la existencia de mutaciones y su localización genómica, pudiendo de esta manera obviar los incovenientes de la inmunohistoquímica.

Las series publicadas en la actualidad sobre la implicación pronóstica de la p53 sobre el carcinoma epidermoide de cabeza y cuello podemos dividirlas en series radioterápicas y quinúrgicas. En las series radioterápicas se han publicado 9 trabajos en los cuales sólo la mitad han podido correlacionar la sobreexpresión de la p53 con el pronóstico. La serie más grande de estos trabajos, con 101 pacientes, es la de RaybaundDiogene, ${ }^{15}$ en la cual encuentra una asociación importante entre la sobreexpresión inmunohistoquímica de la p53 y pronóstico, mayor que la de otros factores clásicos como el T. Sin embargo, series con secuenciación completa del p53 en carcinoma de laringe no encuentran asociación entre las mutaciones y el pronóstico. ${ }^{16}$

Las series quírurgicas son más numerosas; en los estudios multivariantes se presentan aproximadamente un $50 \%$ con correlación pronóstica y la otra mitad sin correlación. La serie con más casos es la de Pruneri, ${ }^{17}$ con 149 pacientes, la mayoría con los cuellos negativos y estadios precoces, en los cuales el $50 \%$ de los pacientes presentaron hipercaptación inmunohistoquímica para la p53. Sin embargo, los resultados no demostraron correlación pronóstica. Analizando el genotipo de la p53 en serie quirúrgicas encontramos el trabajo de Koch, ${ }^{18}$ con 110 casos, con tumores avanzados muchos de ellos. Los resultados evidenciaron una fuerte correlación con la mutación de la p53 en los exones 5 a 9 y la recurrencia locorregional y el pronóstico.

Los autores del presente trabajo presentan unos resultados que se encuadrarían con aquellos con los sí asocian la p53 con el pronóstico a largo plazo. No obstante, los resultados hay que mirarlos como en una serie pequeña de pacientes que no son consecutivos y presentan un tratamiento no estandarizado. El vaciamiento de cuello en cuellos N0, ya que cogen estadios I y II, lo realizan sin un claro criterio, al igual que la radioterapia postoperatoria, lo cual entorpece la interpretación de los resultados al ser una serie de no muchos pacientes. Así mismo, presentan un caso, $\mathrm{n}$ 34 , con una recidiva local al mes del tratamiento que sugiere más que recidiva una persistencia tumoral. Llama la atención que más de la mitad de las recidivas sean cervicales, incluyendo casos con vaciamiento cervical y radioterapia, aunque la mayoría sin tratamiento en el cuello. La presencia de las metástasis cervicales antes del año del tratamiento primario, parece indicar la existencia de metástasis subclínicas en el momento del tratamiento inicial. Todos estos datos pueden hacer pensar que la metodología de tratamiento puede ser un factor determinante en el resultado final de la supervivencia en la presente serie. En conclusión, la p53 parece tener gran utilidad en otros tumores pero en cáncer de cabeza y cuello los resultados contradictorios con inmunohistoquímica limitan su uso en la clínica para la determinación de pronóstico de los pacientes. El uso de técnicas de secuenciación ha sido poco frecuente en nuestros tumores y los resultados no son unánimes; por otro lado, las técnicas de secuenciación no parecen actualmente al alcance de la rutina hospitalaria. Habrá que buscar métodos que permitan obtener resultados más homogéneos o buscar otros marcadores que permitan obtener resultados más repreducibles como la Ciclina D1, Syndecan, la PKC o nuevos marcadores por determinar. Estudios como el presente nos debe animar a seguir esta línea de trabajo y realizar estudios sobre p53 y otros marcadores.

\section{Martínez Gimeno}

Servicio de Cirugía Oral y Maxilofacial Hospital Ntra. Sra. de la Candelaria, Tenerife.

(España) 


\section{Bibliografía}

1. Martínez Gimeno C, García Sabrido Jl, Navarro Vila C. Conceptos básicos actuales sobre la etiología, diagnóstico y tratamiento del cáncer. Bases para la cirugía oncológica. Rev Esp Cir Oral Maxilofac 1989;11:57-67.

2. Quon H, Liu FF, Cummings BJ. Potencial molecular prognostic markers in head and neck squamous cell carcinomas. Head Neck 2001;23:147-15.

3. Francchiolla NS, Pignataro L, Capaccio P, Trecca D, Boletini A, Ottaviani A, Polli E, Maiolo AT, Neri A. Multiple genetic lesions in laryngeal squamous cell carcinomas. Cancer 1995;75:1292-301.

4. Walther EK, Dahlmann N, Gorgulla HT. Tumor markers in the diagnosis and follow up of head and neck cancer. Role of CEA, CA 19-9, SCC, TK and dTTPase. Head Neck 1993;15:230-5.

5. Williams ME, Gaffey MY, Weiss LM, Wilczynski SP, Shuuring E, Lenine PA. Chromosome 11q13 amplification in head and neck squamous cell carcinoma. Arch Otolaryngol Head Neck Surg 1993;119:1238-43.

6. O-charoenrat P, Rhys-Evans P. Expression of vascular endothelial growth factor family members in head and neck squamous cell carcinoma correlates with lymph node metastasis. Cancer 2001;92:556-68.

7. Dietz A, Rudat V, Conradt C, Weidauer H, Ho A, Moehler T. Pronostic relevance of serum levels of the angiogenic peptide bFGF in advanced carcinoma of the head and neck treated by primary radiochemotherapy. Head Neck 2000;22:666-73.

8. Pignataro L, Pruneri G, Carboni N. Clinical relevance of cyclin D1 protein overexpresión in larygeal aquamous cell carcinoma. J Clin Oncol 1998;16:3069-77.

9. Bova RJ, Quinn DI, Nankervis JS. Cyclin D1 an p16INK4a expression predict reduced survival in carcinoma of anterior tongue. Clin Cancer Res 1999;5:2810-9.

10. Michalides RJ, Van-Veelen NM, Kristel PM, Hart AA, Loftus BM, Hilgers FJ, Balm AJ. Arch Otolaryngol Head Neck Surg 1997;123:497-502.
11. Pulkkinen JO, Penttinen M, Jalkanen M, Klemi P, Grenman R. Syndecan-1. A new prognostic marker in laryngeal cancer. Acta Otolaryngol Stockh 1997;117:312-5.

12. Inki $P$, Josensuu $H$, Gernman $R$, Klemi $P$, Jalkanen $M$. Association between syndecan 1 expression and clinical outcome in squamous cell carcinoma of the head and neck. Br J Cancer 1994;70:319-23.

13. Tartour E, Deneux L, Mosseri V, Jaulerry C, Brunin F, Point D, Validire P, Dubray B, Fridman WH, Rodriguez J. Soluble interleukin-2 receptor serum level as a predictor of locorregional control and survival for patients with head and neck carcinoma. A result of a multivariate prospective study. Cancer 1997;79:1401-8.

14. Martínez Gimeno C, Díaz Meco MT, Domínguez I, Moscat J. Alterations in levels of different protein kinase $\mathrm{C}$ isotypes and influence on behavior of squamous cell carcinoma of the oral cavity. $\varepsilon$ PKC, a novel prognostic factor for relapse and survival. Head Neck 1995;17:516-25.

15. Raybaund-Diogene H, Fortin A, Morency R, Roy J, Monteil RA, Tetu B. Markers of radioresistance in squamous cell carcinomas of the head and neck. A clinicopathologic and immunohistochemical study. J Clin Oncol 1997; 15:1030-8.

16. Saunders ME, McKenzie R, Shipman R, Fransen E, Gilbert $\mathrm{R}$, Jordan RC. Pattems of p53 gene mutations in head and neck cancer. Full-length gene secuencing and results of primary radiotherapy. Clin Cancer Res 1999;5:24552463.

17. Pruneri G, Pignataro L, Carboni N. Clinical relevance of p53 and bcl-2 protein overexpression in laringeal squamous cell carcinomas. Int J Cancer 1998;79:263-8.

18. Koch Wm, Brennan JA, Zahurak M. p53 mutation and locorregional treatment failure in head and neck squamous cell carcinoma. J Natl Cancer Ints 1996:1580-6.

19. Schliephake H. Pronostic relevance of molecular markers of oral cancer. A review Int J Oral Maxillofac Surg 2003;32:233-45. 